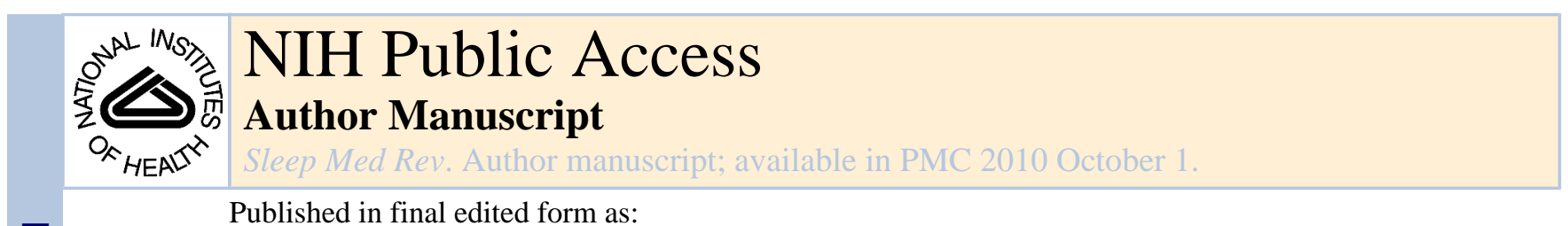

Published in final edited form as:

Sleep Med Rev. 2009 October ; 13(5): 305-308. doi:10.1016/j.smrv.2009.05.004.

\title{
How Do I Remember? Let Me Count the Ways
}

\author{
Robert Stickgold \\ Center for Sleep and Cognition Beth Israel Deaconess Medical Center 330 Brookline Ave / FD-861 \\ Boston MA 02215 USA
}

\section{Introduction}

In this issue, Diekelmann, Wilhelm and Born ${ }^{1}$ provide an extensive and critical review of the rapidly growing literature on sleep-dependent memory consolidation. But rather than simply reviewing this literature, they have taken this opportunity to ask several important questions concerning off-line memory processing. Most gratifying is their decision not to simply look ever more deeply into frequently discussed questions, but rather to raise several new ones. They ask what kinds of memories are consolidated by sleep, what kinds of consolidation are accomplished, and what kinds of sleep are required. For the reader looking for a quick set of answer, they won't find them here or in the Diekelmann article. But the authors have done a superb job of organize the literature to support a series of proposed answers, and I will offer a few more, all of which will hopefully serve as a basis for further investigations.

The task of sorting out the interactions between sleep and memory is a daunting one. We summarized some of these issues in an earlier review. ${ }^{2}$ At the very least (Fig 1), one has to address the question of how five stages of sleep ${ }^{3}$ interact with at least six types of memories ${ }^{4}$ and six stages of post-encoding memory processing, ${ }^{2}$ for a combined total of 144 distinct sub-questions (e.g., how does REM sleep affect the stabilization of classical conditioning; Fig 1). But Diekelmann and colleagues have now added another layer of more subtle questions - how do the strength of initial encoding and the perceived value of the memory interact with these sleep-dependent processes, how do the amount of sleep and the timing of the sleep affect them, and how do these processes change with age and in the presence of psychiatric disorders?

Asking such a wide range of questions allows Diekelmann et al. ${ }^{1}$ to propose broad principles that arguably apply to all forms of sleep-dependent memory processing. Below, I will take issue with some of their proposals, but not because I'm convinced that they're wrong. Rather I am using their excellent review as a platform from which to begin a discussion that will undoubtedly continue across many years of further research.

\section{Definition of memory consolidation}

Diekelmann et al. ${ }^{1}$ define memory consolidation as a collection of processes by which "fresh memory traces are strengthened and transformed into a more stable and persistent form and are integrated into preexisting knowledge networks". ${ }^{1}$ While my definition would be slightly

(C) 2009 Elsevier Ltd. All rights reserved.

(v) 1-617-667-8485 (f) 1-617-667-8498 (e) rstickgold@ @ms.harvard.edu

Publisher's Disclaimer: This is a PDF file of an unedited manuscript that has been accepted for publication. As a service to our customers we are providing this early version of the manuscript. The manuscript will undergo copyediting, typesetting, and review of the resulting proof before it is published in its final citable form. Please note that during the production process errors may be discovered which could affect the content, and all legal disclaimers that apply to the journal pertain. 
different, what is important is that writers clearly define this term in their papers, as there is considerable disagreement as to whether all of these processes are rightfully termed memory "consolidation" or, conversely, whether the term might even refer to additional processes, such as reconsolidation or the extinction of classical conditioning. for human memory, Personally, I have begun using the phrase "memory evolution" to refer to all post-encoding memory processing in humans that occurs without intent or conscious awareness. Such processing is known to occur over time intervals ranging from minutes to decades (Fig 2).

\section{Memory categories}

Diekelmann et al. ${ }^{1}$ argue that post-learning sleep enhances consolidation of declarative, procedural and emotional memories, with emotional memories perhaps more accurately seen as a specific subset of declarative memories. I would argue that this list can actually be expanded. There is now evidence that sleep also facilitates processing of classical conditioning, 5 which, like procedural learning, is a form of nondeclarative memory. Likewise, studies suggest that sleep also facilitates the semantization of episodic memory, transforming detailed episodic memories into more general semantic memories. ${ }^{6}$ In fact, it seems reasonable to propose that sleep benefits all classes of memory, albeit in various ways and to varying degrees. At this point there is no category of memory for which there is strong evidence that sleep provides no benefit.

Diekelmann et al. take a different stance, and offer the provocative hypothesis that "access to sleep-dependent consolidation requires memories to be encoded under control of prefrontalhippocampal circuitry". ${ }^{1}$ They argue that procedural learning task which have shown sleepdependent consolidation in reality occur with explicit awareness of the learning and hence with a declarative (i.e., hippocampal) component. While this is generally the case, it only demonstrates that we cannot rule out a hippocampal requirement for consolidation of these memories.

To strengthen their claim, they point to the work of Robertson et al., ${ }^{7}$ who investigated timeand sleep-dependent enhancement of learning following training on a serial reaction time task. In this task, subjects press four keys, on at a time, matching the order of visually presented stimuli. Testing involves hundreds of stimuli and button presses. Importantly, the order of the stimuli is not random, for example following a repeating $12 \mathrm{key}$ sequence. Robertson $\mathrm{et} \mathrm{al}^{7}$ demonstrated that subjects improved more on the task following a night of sleep than after an equal amount of daytime wakefulness, but only when they were informed prior to training that a repeating pattern was present. When subjects were not informed of this fact, and remained unaware of the sequence's presence, sleep offered no advantage over wake. But the absence of an advantage was not because subjects failed to improve overnight. Rather, it was because equivalent improvement was seen across the day. In fact, there was nonsignificantly more overnight improvement in the non-hippocampal condition (Fig 3).

But stronger evidence of processing without hippocampal mediation is the finding that densely amnesic patients with bilateral hippocampal damage still show normal sleep-dependent consolidation on a visual texture discrimination task, despite the inability of subjects to recall or even recognize the test when retested. ${ }^{8}$ Thus, at least for this one task that has repeated shown sleep-dependent consolidation, ${ }^{9}$ hippocampal involvement is not necessary.

\section{Memory strength, reward, and relevance}

Diekelmann et al. ${ }^{1}$ propose in their review that enhancement is greater for weakly encoded memories than for strongly ones. While several studies that varied the strength of encoding support this conclusion, the authors note that other studies, which looked at normal variation in performance across subjects, found the opposite result, that only those who encoded 
memories more strongly showed subsequent sleep-dependent benefits. My own suspicion is that there is an inverted-U shaped curve of sleep benefit (Fig 4). This would result in both very weak and very strong memories failing to benefit from sleep, while those with intermediate levels of initial encoding would show the greatest benefit. As a result, some tasks would show enhanced sleep-dependent consolidation with stronger encoding would (Fig 4, left hand ellipse and arrow), while others would show more benefit with weaker initial encoding (Fig 4, right hand ellipse and arrow).

\section{Sleep timing, duration, and stages}

Diekelmann et al. ${ }^{1}$ provide an excellent review of the impact of sleep timing, duration, and stages on consolidation, and offer several hypotheses as to how each of these factors contribute to the extent of sleep-dependent consolidation. But I think the issues surrounding naps need comment. There is something about naps that we fundamentally fail to understand. As little as 6 minutes of sleep has been reported to produce a significant $18 \%$ increase in subsequent verbal recall (compared to controls who remained awake), and a 25 min nap produced almost twice as much improvement. ${ }^{12}$ These benefits are equal to or even larger than those seen from a full night of sleep on similar verbal memory tasks $\left(e . g .{ }^{6}\right)$. The same accelerated benefits of naps has been reported for both visual perceptual and motor skill tasks, where 60-minute nap opportunities again produced as much improvement as a whole night of sleep. ${ }^{13},{ }^{14} \mathrm{Lahl}$ and colleagues ${ }^{12}$ suggested that sleep was only necessary to initiate a process of consolidation that could then continue in its absence. But studies of perceptual and motor skill improvement found that the extent of post-nap improvement correlated with the sleep-stage distribution during the intervening nap, ${ }^{13}, 14$ suggesting that improvement actually depended on the course the nap took. It thus appears that there remains a major conceptual gap in our thinking about how naps facilitate memory consolidation.

How specific sleep stages relate to these processes remains an area of vigorous investigation. Diekelmann et al. ${ }^{1}$ argue for the hypothesis that SWS is most important for consolidation of declarative memories, while REM is more important for procedural learning. There is certainly a large body of data to support this model. But as they acknowledge, there is a considerable body of findings that would seem to contradict it.

I would like to suggest an orthogonal model for the role of different sleep stages, namely that sleep-stage specificity exists not for categories of memories (Fig 1B), but for stages in the consolidation process (Fig 2). According to such a model, SWS serves to stabilize recently acquired declarative and procedural memories through a process of synaptic level consolidation that maintains and stabilizes synaptic modifications produced by initial memory encoding. ${ }^{15}$

REM and Stage 2 NREM sleep subsequently facilitates systems level consolidation processes that enhance the efficacy of these memories. ${ }^{16}$ Thus, while SWS serves the classical function of consolidation, namely memory stabilization, REM and Stage 2 NREM support a variety of powerful sleep-dependent memory processes that act to $(i)$ enlarge the neural networks in which memories are stored, (ii) integrate these new memories with other, older memories, into rich semantic networks, and (iii) extract patterns and rules from large bodies of encoded information.

It is important to reiterate that this model is largely orthogonal to the one proposed by Diekelmann et al. ${ }^{1}$ The design of the vast majority of sleep-dependent declarative memory tasks so far studied essentially measure the extent of synaptic level consolidation, and hence both models predict a SWS dependence. Similarly, enhancement of procedural task learning is accompanied by absolute enhancement of performance and systems level reorganization of the memories, ${ }^{17}, 18$ and thus both models predict improvement based on REM or Stage 2 NREM sleep. 


\section{Conclusion}

Diekelmann et al. ${ }^{1}$ have provided a detailed review and conceptual analysis of the literature on sleep-dependent memory consolidation in humans. Based on their review, they have offered a number of viable and testable hypotheses concerning how this consolidation is produced, as well as how it is regulated and limited. These hypotheses can now form the basis for further hypothesis generation and testing. I have offered above some directions in which such extensions might go are.

In concluding, I would like to comment on two questions that Diekelmann et al.\{Diekelmann, 2009 \#6157\} touched on only briefly. First, how sophisticated are the algorithms that control how memories are processed during sleep? At one extreme, the algorithm could be very simple, calculated at the level of individual synapses, based on each synapse's physical and chemical structure. Tononi and Cirelli's model of synaptic homeostasis ${ }^{19,} 20$ is an example of a mechanism by which such consolidation could occur, although it would arguably consolidate new and pre-existing cortical memories equally. Still, minor modifications could allow for recently formed memories to be selectively enhanced based on the unique chemical signatures of their synapses.

At the other extreme, we have proposed ${ }^{6,11}$ that the selection of memories to consolidate and the determination of the form of that consolidation, reflect complex, sophisticated, and nuanced mechanisms. This would help explain the apparently contradictory findings concerning the impact of strength of initial encoding described by Diekelmann et al., ${ }^{1}$ as well as their finding that motivational factors, such as financial reward for better performance, increases sleepdependent consolidation. It would also fit with more recent findings that emotional elements within memorized scenes benefit selectively from sleep, ${ }^{11}$ and that recall of words which describe the gist of a studied wordlist are preferentially recalled after sleep, even when they are not actually present in the studied list. ${ }^{6}$ Such mechanisms would presumably invoke systems level consolidation processes. Taken as a whole, this argument leads to the hypothesis that the sleeping brain identifies those recent memories, or even those components or associates of recent memories, that would be most usefully maintained, strengthened, and integrated during sleep, and selectively consolidates them. From this perspective, sleep-dependent memory consolidation could be construed as the sleep-state equivalent of Piaget's theory that children are most interested in the "moderately novel", because this is where investigation by the child is most informative. ${ }^{10}$

The second question I want to raise is how and when memories are "tagged" for subsequent processing. Such tagging could occur at the time of encoding, possibly based on the strength of the initial encoding or on the simultaneous presence of affect. More intriguingly, however, is the possibility that tagging results from more complex analyses performed subsequent to initital encoding. For example, they could be tagged during presleep hypnagogic thought processes, which seem to review emotional memories from the preceding day; or they could be selected at the time of consolidation based on their ease of reactivation during one sleep stage or another; or they could even be tagged and identified by their reactivation during dreaming. For now, all of these possibilities are on the table.

\section{Acknowledgements}

This work was supported by grant MH48,832 from the National Institutes of Health (USA).

\section{References}

1. Diekelmann S, Wilhelm I, Born J. The whats and whens of sleep-dependent memory consolidation. Sleep Med Rev. 2009in press 
2. Walker MP, Stickgold R. Sleep-dependent learning and memory consolidation. Neuron Sep 30;2004 44(1):121-33. [PubMed: 15450165]

3. Rechtschaffen, A.; Kales, A. A manual of standardized terminology, techniques and scoring system for sleep stages of human subjects: Brain Information Service. University of California; Los Angeles: 1968.

4. Tulving E. How many memory systems are there? American Psychologist 1985;40:385-98.

5. Pace-Schott EF, Milad MR, Orr SP, Rauch SL, Stickgold R, Pitman RK. Sleep promotes generalization of extinction of conditioned fear. Sleep Jan 1;2009 32(1):19-26. [PubMed: 19189775]

6. Payne JD, Schacter DL, Propper RE, Huang L-W, Wamsley EJ, Tucker MA, et al. The role of sleep in false memory formation. Neurobiol Learn Mem. 2009

7. Robertson EM, Pascual-Leone A, Press DZ. Awareness modifies the skill-learning benefits of sleep. Curr Biol Feb 3;2004 14(3):208-12. [PubMed: 14761652]

8. Stickgold, R. Human studies of sleep and off-line memory reprocessing. In: Maquet, P.; Smith, C.; Stickgold, R., editors. Sleep and Plasticity. Oxford University Press; New York: 2003. p. 41-63.

9. Stickgold R, Whidbee D, Schirmer B, Patel V, Hobson JA. Visual discrimination task improvement: A multi-step process occurring during sleep. J Cogn Neurosci 2000;12:246-54. [PubMed: 10771409]

10. Piaget, J. The origins of intelligence in children. International Universities Press, Inc.; New York: 1952.

11. Payne JD, Stickgold R, Swanberg K, Kensinger EA. Sleep preferentially enhances memory for emotional components of scenes. Psychol Sci 2008;19:781-8. [PubMed: 18816285]

12. Lahl O, Wispel C, Willigens B, Pietrowsky R. An ultra short episode of sleep is sufficient to promote declarative memory performance. J Sleep Res Mar;2008 17(1):3-10. [PubMed: 18275549]

13. Mednick S, Nakayama K, Stickgold R. Sleep-dependent learning: a nap is as good as a night. Nat Neurosci 2003;6:697-8. [PubMed: 12819785]

14. Nishida M, Walker MP. Daytime naps, motor memory consolidation and regionally specific sleep spindles. PLoS ONE 2007;2(4):e341. [PubMed: 17406665]

15. Wang H, Hu Y, Tsien JZ. Molecular and systems mechanisms of memory consolidation and storage. Prog Neurobiol Jun;2006 79(3):123-35. [PubMed: 16891050]

16. Stickgold R, Walker MP. Sleep-dependent memory consolidation and reconsolidation. Sleep Med Jun;2007 8(4):331-43. [PubMed: 17470412]

17. Walker MP, Stickgold R, Jolesz FA, Yoo SS. The functional anatomy of sleep-dependent visual skill learning. Cerebr Cortex 2005;15:1666-75.

18. Walker MP, Stickgold R, Alsop D, Gaab N, Schlaug G. Sleep-dependent motor memory plasticity in the human brain. Neuroscience 2005;133(4):911-7. [PubMed: 15964485]

19. Tononi G, Cirelli C. Sleep function and synaptic homeostasis. Sleep Med Rev Feb;2006 10(1):49_ 62. [PubMed: 16376591]

20. Tononi G, Cirelli C. Sleep and synaptic homeostasis: a hypothesis. Brain Res Bull Dec 15;2003 62 (2):143-50. [PubMed: 14638388]

21. Stickgold R, Walker MP. Memory consolidation and reconsolidation: what is the role of sleep? Trends Neurosci 2005;28(8):408-15. [PubMed: 15979164] 

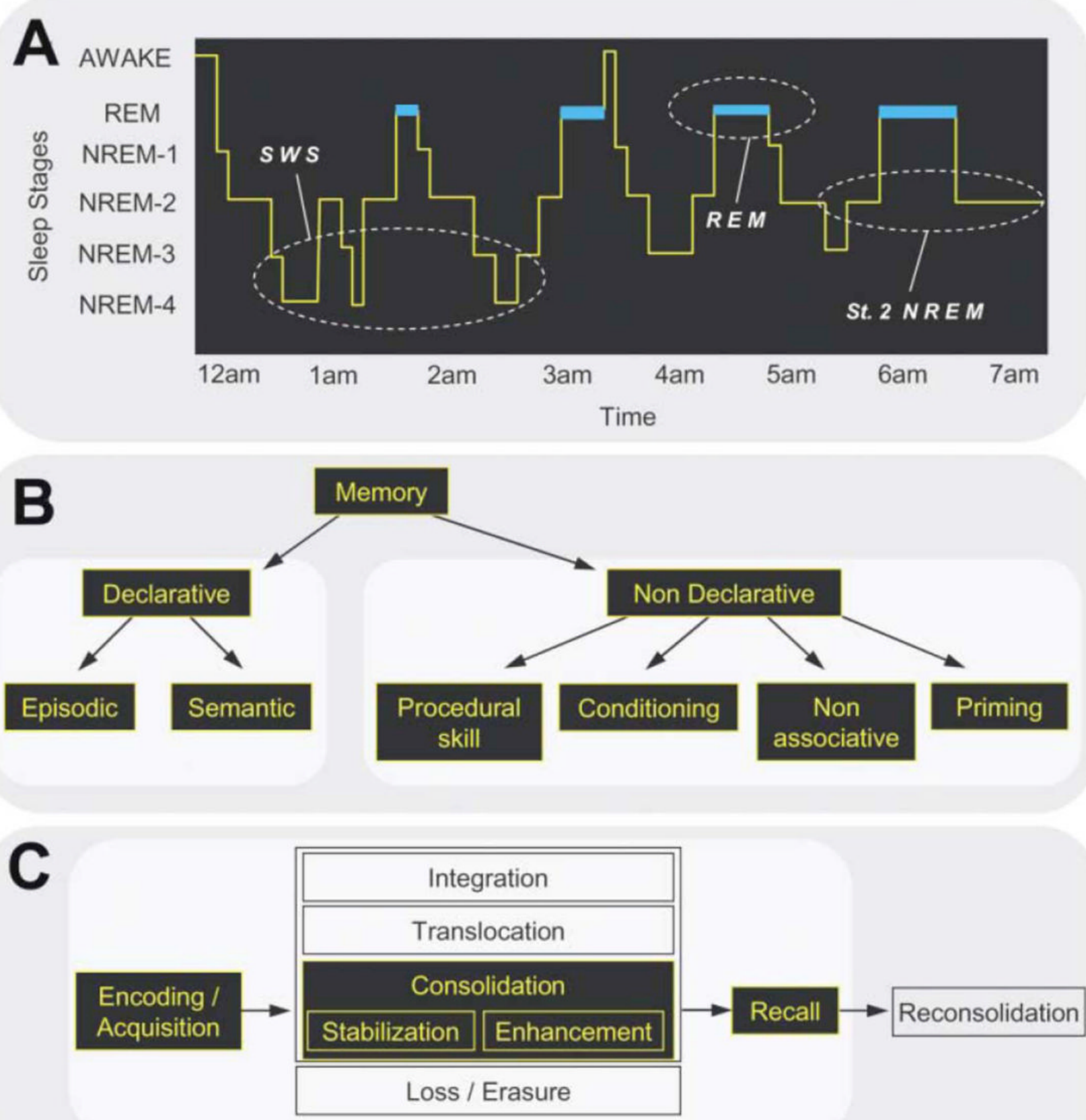

Figure 1. The sleep cycle, memory systems, and memory stages

(A) The human sleep cycle - across the night, NREM and REM sleep cycle every 90 min in an ultradian manner, while the ratio of NREM to REM sleep shifts. During the first half of the night, stages 3 and 4 NREM (SWS) dominate, while stage 2 NREM and REM sleep prevail in the latter half of the night. EEG patterns also differ significantly between sleep stages, with electrical oscillations such as K complexes and sleep spindles occurring during stage 2 NREM, slow $(0.5-4 \mathrm{~Hz})$ delta waves developing in SWS, and theta waves seen during REM. (B) Memory systems - human memory is most commonly divided into declarative forms, with further subdivisions into episodic and semantic; and nondeclarative forms, subdivided into an array of different types including procedural skill memory (C) Developing stages of memory 
- following the initial encoding of a memory, several ensuing stages are proposed, beginning with consolidation, as well as integration of the memory representation, translocation of the representation, or erasure of the memory. Also, following later recall, the memory representation is believed to become unstable once again, requiring periods of reconsolidation. Reproduced from 2. 


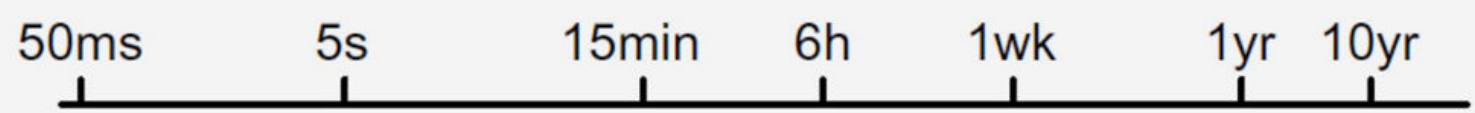

\section{Encode}

\section{Stabilize}
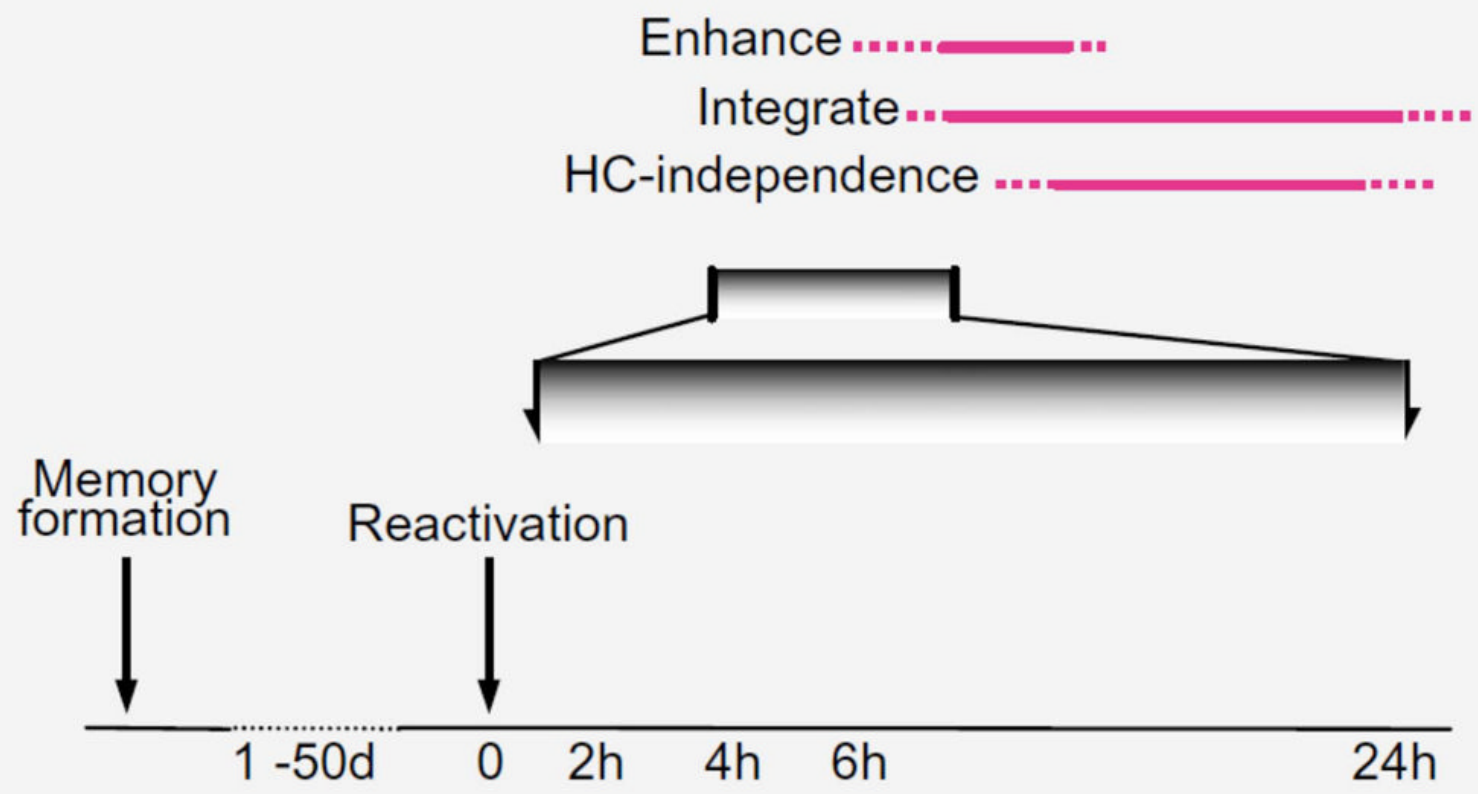

Destabilize

Deteriorate.........

Reconsolidate

Figure 2. Time course of memory processes

(a) Memory formation and consolidation. After the initial rapid encoding of a sensory experience, the neural representation of the memory can go through several automatic processes, independent of rehearsal, intent or awareness. These can stabilize and enhance a memory, so it is resistant to interference and more effective to guiding behavior, and can also integrate the memory into larger associative networks. The latter process is thought to permit episodic memories to be recalled without hippocampal (HC) involvement. The extent to which such processes affect different memory systems is unclear. Note the logarithmic time scale. (b) Memory reactivation and reconsolidation (featuring an enlargement of (a), as indicated by the gray shading). After stabilization is complete, reactivation of a memory can lead to its return to an unstable form. Normally, such memories appear to be reconsolidated following this destabilization but, if such reconsolidation is blocked, degradation of the memory can ensue (see also Figure 4). Solid bars represent periods of known processing; dotted bars reflect hypothesized or variable periods of processing. Reproduced from 21 . 

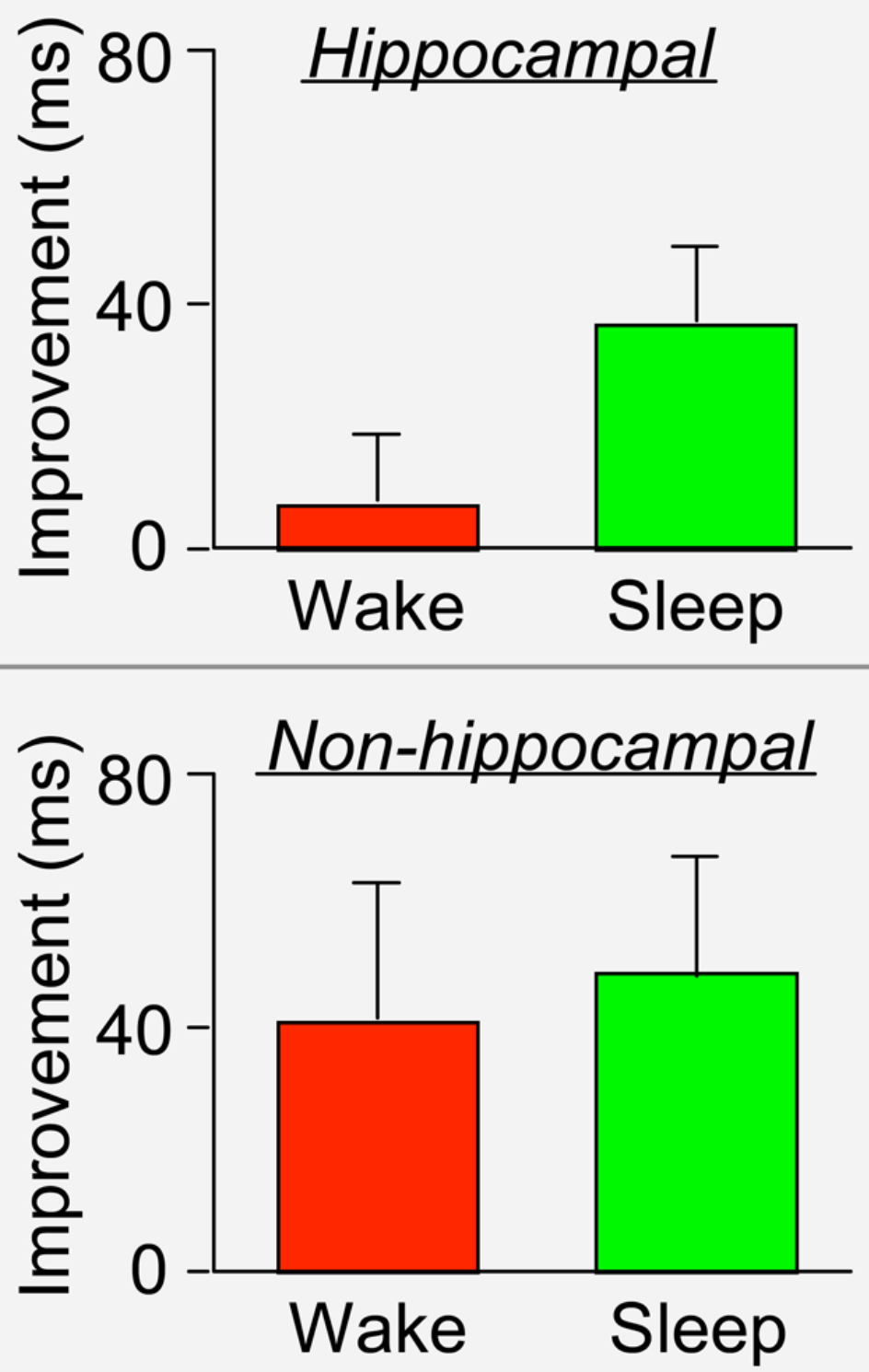

Figure 3. Time-dependent and sleep-dependent improvement on serial reaction time task Subjects trained at either 8AM (Wake) or 8PM (Sleep) and were retested $12 \mathrm{hr}$ later. Top: Hippcampally dependent learning - subjects were informed of the presence of the repeating response sequence prior to training; Bottom: Non-hippocampally mediated learning - subjects were not informed of the sequence, and did not become aware of it during training. Modified from 7. 


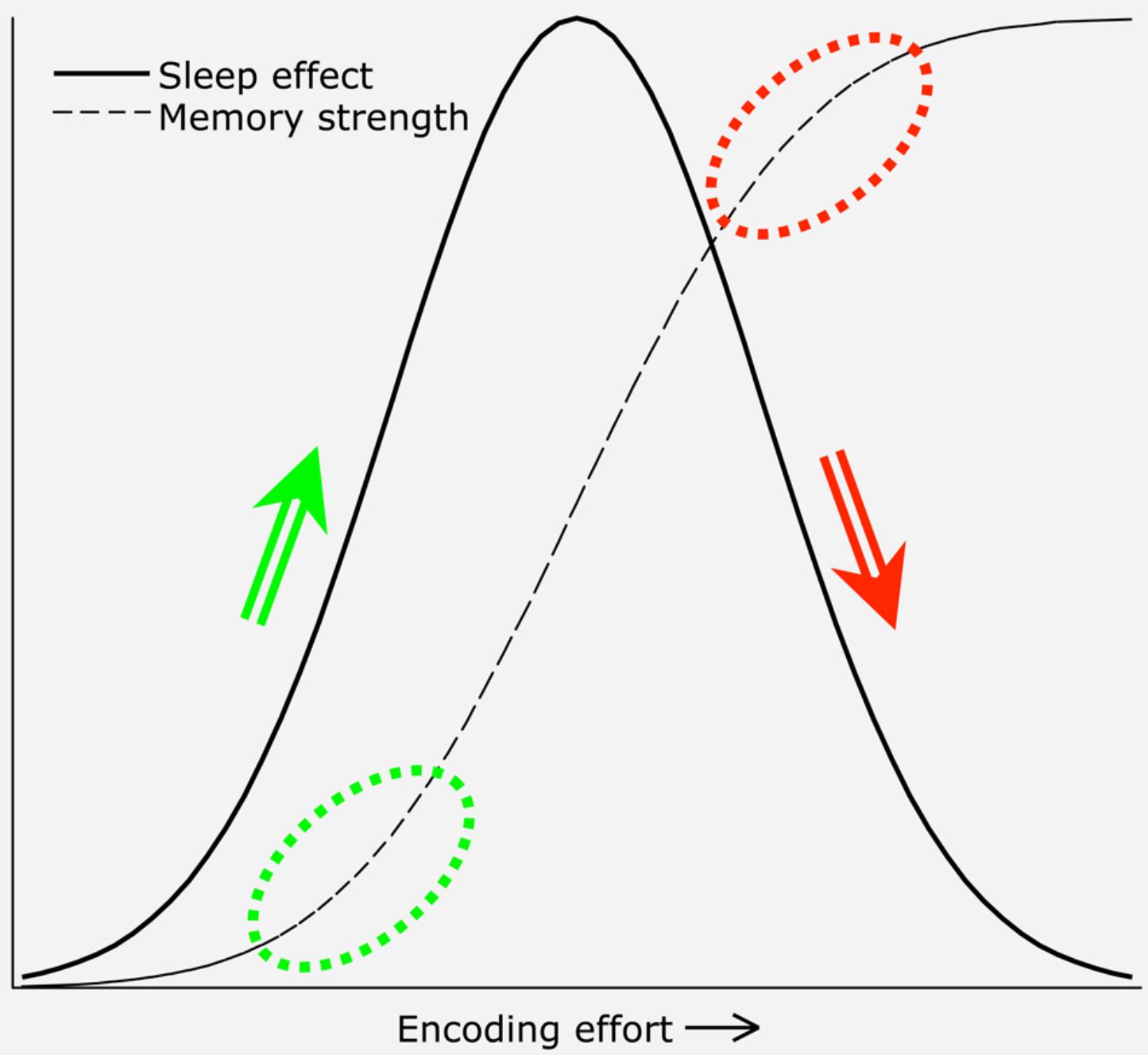

Figure 4. Logistic curve for memory strength

The extent of sleep-dependent enhancement/consolidation of a memory (solid line) depends on its initial strength (dashed line), being maximal and intermediate strengths. Ellipses indicate regions of the memory strength curve where greater initial strength leads to either increases (on left) or decreases (on right) in the extent of subsequent sleep-dependent consolidation, as depicted by arrows. 